\title{
Gobierno universitario: entre la autogestión estamental y la responsabilidad social
}

Josep M. Vallés *

El argumento que se desarrolla en este trabajo ${ }^{1}$ gira en torno a tres conceptos: autonomía, responsabilidad social -expresada en rendición de cuentas o accountability- y gobernabilidad. Son tres conceptos que se presentan estrechamente vinculados cuando se analiza la viabilidad futura de una amplia gama de instituciones y agencias públicas, sujetas a crecientes exigencias de eficiencia y calidad en un nuevo contexto socioeconómico.

Las Universidades públicas son un buen ejemplo de ello. Aparecen como instituciones al servicio de la comunidad. Han ido alcanzando un grado considerable de autonomía interna. Están sujetas a una creciente exigencia de resultados por parte de su entorno social, económico y político. Por todo ello, establecer la relación adecuada entre autonomía, responsabilidad social y capacidad de gobierno constituye uno de los retos más importantes -si no el más importante- de los que tiene hoy planteados la Universidad pública ${ }^{2}$. En el caso español, la experiencia acumulada desde la vigencia de la LRU -como elemento central de una nueva política universitaria-, permite extraer algunas lecciones y aventurar algunas propuestas.

\section{La transformación del} sistema universitario español: crecimiento acelerado $\mathrm{y}$ desregulación parcial

A semejanza de lo ocurrido en la mayor parte de los sistemas europeos, el sistema universitario espanol ha experimentado en las dos últimas décadas un proceso de cambio, caracterizado por la expansión cuantitativa, la creciente complejidad en la definición y provisión de sus servicios y la relativa desregulación de sus operaciones.
No entramos en la descripción detallada del proceso espanool y de sus singularidades. Señalaremos, únicamente, que -a diferencia de otros países - se ha producido en un período temporal más breve y condensado, acumulando las dificultades que otros sistemas han ido afrontando en etapas sucesivas.

Nuestra atención se concentra en uno de los aspectos de la transformación general del sistema: la desregulación parcial contenida en la LRU y en las medidas y disposiciones adoptadas en su aplicación. Este proceso de desregulación incide sobre dos aspectos. Por una parte, afecta a la coordinación del sistema universitario público en su conjunto. Por otra, se expresa en la definición del autogobierno de la institución universitaria. Tanto el primero como el segundo pueden entenderse como manifestaciones de un principio de autonomía universitaria, declarado formalmente en la Constitución de 1978 y cuya caracterización jurídica ha sido objeto de controversia que no examinamos aquí ${ }^{3}$.

Sobre la coordinación general del sistema universitario público, baste decir que la LRU apunta a una reducción del papel regulador tradicional del Estado $\longrightarrow$ de la correspondiente Administración territorial de tutela - en beneficio de la propia comunidad universitaria y, en menor medida, de los agentes sociales o de un cierto "cuasi-mercado".

En una bien conocida tipología, se han clasificado los modelos de coordinación general del sistema universitario, según su principal agente coordinador ${ }^{4}$. Los tres tipos-ideales presentan, respectivamente, al Estado, a la propia comunidad académica y a la "Sociedad" o a un teórico "mercado" como actores dominantes en la regulación-coordinación.

En este triángulo ideal, la posición tradicional del sistema español -junto a otros países europeos continentales de tradición centralista - se situaba claramente muy cerca del vértice "Estado". La LRU ha significado, con sus cambios, un desplazamiento muy moderado hacia el vértice asociedad-mercado" -donde se situaría teóricamente un modelo anglo-americanoy, de manera algo más acusada, una aproximación al vértice 
"Comunidad académica" -que podrían ser determinadas versiones de la Universidad germánica-.

Este incremento de capacidad reguladora interna que se deja en manos de la propia comunidad académica pone inmediatamente sobre la mesa el sistema de toma de decisiones de la Universidad, o, en otras palabras, de su sistema de gobierno. En términos normativos, podría decirse que el incremento de la autonomía universitaria reclamaría un aumento en la gobernabilidad de la institución, entendida como adecuación entre las decisiones adoptadas por la institución y las demandas y expectativas que provienen de su entorno.

Desde esta perspectiva pierde sentido la polémica sobre el "mayor o menor" grado de autonomía universitaria contenido en la LRU. La coordinación del sistema, en definitiva, es resultado de una acción combinada de una variedad de actores, entre los que ha ganado influencia la propia comunidad académica. Recordemos además, a este respecto, que la etiqueta “Estado" -o cualquier otro tipo de actor público- recubre frecuentemente la propia confesión académica o, más exactamente, determinados sectores de la misma ${ }^{5}$.

\section{Autonomía y autogobierno en la LRU}

Desde esta perspectiva "desreguladoran, ¿qué modelo de autogobierno universitario definió la LRU? En términos generales, la LRU - hija de su tiempo y de la vivencia generacional de quienes la promovimos y aprobamos- reaccionaba contra el patrón jerárquico y verticalista de la Universidad del franquismo. En claro antagonismo con este modelo, convertía una idea determinada de la participación democrática en criterio exclusivo de legitimación de todas las decisiones internas.

Con ello, se apartaba de otros modelos de gobierno conocidos. En unos casos, los órganos principales de decisión fundan su legitimidad en actores externos a la propia Universidad; en otros, se da una mayor intervención a la propia comunidad universitaria, identificándola generalmente con el profesorado de mayor rango académico.

La LRU optó por un diseño notablemente innovador, cuya arquitectura institucional es bien conocida. Aquí señalamos solamente sus trazos principales:

1) Rigurosa participación estamental -profesorado, personal no docente, estudiantes- en todos los ámbitos (académico, económico, administrativo, etc.) y en todos los niveles de decisión (central, de Facultad o Escuela, de Departamento o Instituto, etc.).
2) Carácter electivo de todos los órganos colegiados y unipersonales de gobierno de la Universidad, tanto en el ámbito central como en el periférico (Departamentos, Facultades, Escuelas, Institutos, etc.), tanto competentes en cuestiones científico-docentes, como de carácter económico-administrativo. La única excepción a la regla de la electividad la constituye el Gerente.

3) Presencia de una representación de los poderes públicos y de los actores sociales en un único órgano: El Consejo Social, órgano de aparticipación de la sociedad en la Universidadn. A este órgano se confían competencias presupuestarias y fiscalizadoras de la actividad universitaria, compartidas o solapadas con las de otros órganos de gobierno.

4) Identificación del Rector como ejecutor de las decisiones de los demás órganos de gobierno y como representante externo de la institución. A su lado y designado por él, el Gerente -incompatible con el desempeño de actividades académicas - es el director de los servicios económicos y administrativos.

5) Finalmente, uniformidad del sistema de gobierno para todas las Universidades públicas, con independencia de sus diferencias en dimensión, composición interna, tradición o voluntad de la propia institución.

Estos criterios generales han dejado escaso margen de variación en la elaboración de los Estatutos de cada Universidad, que -en términos generales- han adoptado esquemas de gobierno bastante parecidos y sin diferencias notables.

De la síntesis anterior queda claro que la LRU se separaba sustancialmente del esquema tradicional de gobierno de las Universidades españolas. Podría afirmarse que se pasaba de un sistema jerarquizado y burocratizado a un modelo autogestionario y estamental, formalmente compensado por una representación sociopolítica externa en el Consejo Social y asistido por la competencia profesional del Gerente.

\section{Un balance de la experiencia LRU}

¿Cómo valorar la experiencia LRU en sus diez años largos de vigencia? Pese a críticas parciales, sería injusto negar que en buena parte de las Universidades españolas ha progresado la profesionalización de su personal, la calidad de sus infraestructuras, la apertura a ciertas demandas del entorno socioeconómico y el nivel científico de su producción investigadora. 
El modelo autogestionario que la Universidad exhibe como excepción llamativa respecto de cualquier otra administración, servicio público u organización privada ha servido probablemente para legitimar determinadas decisiones en un primer período de reformas. Asimismo, ha contribuido a la movilización de energías bloqueadas hasta entonces por una administración rutinaria de la enseñanza superior o especialmente concentradas en la oposición y crítica al régimen político desde el ámbito universitario.

Pero estos resultados globalmente positivos no pueden atribuirse exclusivamente al nuevo sistema de gobierno. Coincidieron con él otros factores de tanta o más importancia que los normativos, entre los que destacamos:

- el crecimiento sostenido de los recursos públicos (económicos, personales) puestos a disposición de las Universidades públicas, especialmente en los años 19851990 , con predominio frecuente de medidas incrementalistas sobre otras consideraciones;

- el voluntarismo de determinados sectores del personal docente y no docente, dispuesto a superar los inconvenientes estructurales del propio sistema de gobierno;

- finalmente, el liderazgo personal de algunos responsables universitarios, dispuestos a desarrollar en el ámbito universitario la vocación de servicio público que otros colegas desarrollaron en el escenario político-institucional.

Estos factores circunstanciales compensaron temporalmente defectos importantes del sistema. Pero esta compensación ha sido menos efectiva, cuando se han congelado o reducido los recursos públicos disponibles o se ha erosionado la disponibilidad voluntarista de grupos y personas, con la emergencia de nuevas condiciones - positivas y negativas- en el ámbito profesional de la carrera académica.

A partir de este momento, se ha hecho patente la debilidad del actual sistema de gobierno universitario. Aparece como poco sensible a nuevas exigencias sociales dirigidas a la Universidad y como poco flexible para adoptar los necesarios cambios que satisfagan aquellas exigencias.

De modo más específico, observadores y operadores del propio sistema han puesto de relieve sus principales inconvenientes, que sintetizamos a continuación:

- la toma de decisiones es demasiado lenta y costosa -en términos políticos y económicos-, en un momento que reclama la adaptación permanente del servicio a nuevas necesidades y cuando más conveniente es disponer de una fuerte capacidad de negociación de la institución ante administraciones y entidades financiadoras, agentes sociales u opinión pública;

- la participación estamental en todos los niveles y ámbitos refuerza de hecho los mecanismos de veto y obstrucción con resultados tendencialmente conservadores. La búsqueda del consenso hace que muchas decisiones respondan a la constitución de coaliciones negativas, más preocupadas por el corto plazo y el interés sectorial que por el medio y largo plazo y el interés global de un servicio público;

- el predominio del esquema colegial difumina las responsabilidades en la adopción de decisiones (o en la no adopción de las mismas), hasta facilitar la impunidad por las consecuencias de cada acción o inacción;

- una visión global del interés de la institución es a menudo relegada a un segundo término, debido al relieve que adquieren los intereses corporativos. Lo manifiesta la tendencia a transformar algunos órganos de gobierno en :mesas paritarias. paralelas de negociación laboral. Igualmente, lo revelan la inasistencia, inhibición o abstención de muchos miembros electivos de los órganos de gobierno cuando se tratan cuestiones que no afectan directamente a su propio estamento ${ }^{6}$;

- el carácter electivo de todos los órganos -colectivos y unipersonales - comporta un elevado coste en términos institucionales y personales: la institución se priva durante un período del rendimiento científico de un grupo de profesores, les exige una dedicación intensa a la gestión para la que no están preparados y —una vez adquirida una cierta competencia en la misma-, les devuelve de nuevo a su tarea académica en inferioridad de condiciones y rendimiento;

- la ambigua posición del Consejo Social —en su composición y competencias- debilita o anula su papel como impulsor de una política institucional global que pueda compensar las dinámicas corporativas internas. Igualmente, dificulta el establecimiento de sistemas efectivos de control y auditoría interna sobre los rendimientos institucionales.

De todo ello se desprende la dificultad del actual sistema para combinar un grado suficiente de autogobierno institucional -como expresión de la autonomía - y la responsabilidad social exigible a un servicio público. Esta deficiente conexión entre autonomía y accountability constituye el nudo del problema de la gobernabilidad.

\section{La Universidad española como «burocracia profesional con vetos estamentales"}

¿Son específicos de la Universidad española los déficit de gobernabilidad que hemos señalado? La respuesta es negativa. 
Tales inconvenientes — con intensidad variable - se registran también en otros modelos universitarios, que comparten características propias de las denominadas aburocracias profesionales*, que por la naturaleza de sus tareas son resistentes a sistemas de gobierno jerárquico ${ }^{7}$.

Pero lo que en algunos análisis era considerado como ventajas de este modelo - señalado incluso como ejemplo para otro tipo de organizaciones - empieza a convertirse en inconveniente cuando cambian rápidamente las demandas del entorno y se renuevan las tecnologías y los procesos de provisión de servicios. Los sistemas de coordinación y control mediante aajustes flexibles" se manifiestan como poco eficientes y ponen sobre la mesa la necesidad de recurrir a diferentes sistemas de coordinación y de gobierno.

Desde esta óptica, el problema central del gobierno universitario en un entorno cambiante y más exigente es el de articular de manera suficientemente efectiva los tres niveles siguientes:

- el nivel primario de las unidades básicas internas, en las que se imparte la docencia y se desarrolla la investigación, en un "cara a cara" permanente con sus usuariosdestinatarios: estudiantes, agencias públicas, empresas, etc.;

- el nivel central, en el que deben adoptarse las decisiones de alcance estratégico que comprometen a la totalidad de la institución universitaria por lo que hace a los objetivos generales, a la distribución interna de los recursos disponibles y al control de su uso eficiente;

- el nivel de coordinación general de todo el sistema público universitario, desde el que se transmiten las exigencias sociales, se determina la intervención pública -financiación, regulación - al servicio de la enseñanza superior y se controlan los resultados obtenidos.

Cuando esta coordinación es deficiente, aparece el problema de la aingobernabilidad universitaria. La necesidad de afrontarlo ha llevado a diversas fórmulas de organización interna, definidas por las autoridades reguladoras o por las propias Universidades. En estos intentos de reforma, se configuran modelos que combinan la colegialidad tradicional de la comunidad académica, el "managerialismo" propugnado por una determinada interpretación de la eficiencia y otras fórmulas varias de participación estamental, más o menos "populistas". Cada modelo establece un cierto equilibrio entre estos componentes, con resultados variables en las diferentes experiencias concretas.

En el caso español, el modelo de la LRU sustituyó un sistema de gobierno centralizado y burocrático por un sistema que podemos calificar como aburocracia profesional con vetos estamentales. En efecto, la configuración peculiar de una abu- rocracia profesionalo, gobernada por la propia comunidad de operadores expertos —en este caso, los profesores-, quedaba matizada por la intervención de otros dos colectivos: personal no docente y estudiantes. Pero, como ya se ha dicho, la aproximación corporativa de estas intervenciones se ha proyectado a menudo sobre el gobierno universitario más como una capacidad de veto que como una capacidad de impulso o de iniciativa para la renovación estratégica, tanto en la respuesta a la demanda social como en la puesta en marcha de mecanismos de rendición de cuentas $\longrightarrow$ accountability-.

\section{Algunas líneas de reforma: funciones de gobierno en los niveles central y periférico de la Universidad}

La dificultad del problema es manifiesta. Como se ha dicho, otros sistemas universitarios han experimentado también cambios en el gobierno interno de la institución universitaria, con resultados discutidos. A partir de estas experiencias y dentro de esta estrategia de exploración de alternativas, se ha elaborado la propuesta que sigue. Se definen en ella funciones, niveles y órganos.

A) En un nivel central debería situarse el desempeño de las funciones que aseguren la coherencia de un proyecto institucional específico para cada Universidad en el marco del sistema universitario público. Esta responsabilidad implica, entre otras funciones, las siguientes:

- Diseño de estrategias gobales a medio/largo plazo de la institución-misión" de la Universidad, uplan estratégico", "planificación plurianual", etc.- entendidos como eventuales expresiones de las grandes líneas de un proyecto global.

- Interacción con los agentes externos (administraciones de tutela, intereses sociales generales; entidades y sujetos financiadores: empresas, antiguos alumnos, fundaciones, etc.) en lo que se refiere a las estrategias globales ya mencionadas.

- Implantación de la estrategia en el aspecto central en la definición de los servicios a proveer (tipos de estudios, grandes áreas de investigación, otros) y en la distribución interna de recursos (inversión física; plantillas de personal, selección de personal y de estudiantes, etc.).

- Elaboración de las regulaciones básicas de carácter general (académicas: admisión y permanencia de estudian- 
tes, reclutamiento de personal docente y no docente; económicas: contratación, régimen de prestaciones, etc.; gestión de recursos humanos, etc.).

- Control de la implantación y evaluación de resultados para las políticas globales, diseñando y supervisando los procesos de control de calidad —centrales y periféricos-, etc.

- Apoyo y asesoramiento a las unidades específicas de producción periférica (estudios/carreras, grupos/institutos de investigación, otros);

- Intervención directa en actividades específicas cuando tienen valor estratégico para el conjunto de la institución (nuevas iniciativas/servicios: nuevas carreras, formación continuada, intercambio internacional, etc.).

B) En el nivel periférico de gobierno se sitúa la adaptación a ámbitos particulares del proyecto global de la institución y la administración de los servicios singulares - programas docentes, de investigación, otros- que le corresponden. En esta tarea se incluye:

- Implementación en el ámbito de su competencia de las decisiones de los órganos generales de gobierno.

- Administración de los recursos asignados - personales, materiales, económicos- para llevar a cabo dicha implementación.

- Participación en el proceso de evaluación de resultados de su ámbito respectivo.

- Propuesta ante los órganos centrales de la modificación de las políticas generales menos eficientes o menos adaptadas a las necesidades directas del servicio y de sus usuarios, participando en la revisión periódica de las estrategias globales a medio y largo plazo.

\section{Una revisión de los órganos de gobierno}

Para el desempeño de las funciones anteriores, se hace aquí una determinada asignación de competencias entre órganos. La propuesta nace de la apreciación crítica del modelo de la LRU y del análisis comparado de otros tipos de gobierno universitario, examinando las ventajas e inconvenientes que se manifiestan en las experiencias correspondientes. Por razones de brevedad, no se exponen aquí los argumentos en favor de la opción que se desarrolla ${ }^{8}$. Advirtamos solamente que la propuesta se hace con plena conciencia de la inexistencia de una alternativa capaz de solventar todos los inconvenientes: se ofrece como material para la crítica y el debate en curso.
1) El nivel central de gobierno.-Las funciones asignadas a un nivel central $\multimap$ global - de la institución corresponderían a un Consejo de Gobierno, como órgano deliberante central, a una Junta Académica y a un Claustro, como órganos consultivos, y al Rector, como órgano ejecutivo general.

En la distribución de competencias, se intentan evitar algunos solapamientos del modelo actual (Consejo Social, Junta de Gobierno, Claustro), se procura deslindar las funciones deliberante, consultiva y ejecutiva de cada órgano y se intenta reducir la deriva estamentalista dominante.

1.1. El Consejo de Gobierno es un órgano colegiado que asume las principales funciones de carácter estratégico, normativo y de control, entre las que figuran las siguientes:

- la definición de la misión y estrategia de la institución;

- la programación plurianual de actividades;

- la aprobación del presupuesto de ingresos y gastos;

- la contratación de obras y servicios;

- la auditoría económica y operativa;

- la aprobación de los Estatutos de la Universidad y de las regulaciones generales en materia de personal, alumnado, etc.;

- la aprobación de las plantillas docentes y no docentes;

- el nombramiento y la revocación del Rector;

- el nombramiento y la revocación del Gerente, a propuesta del Rector;

- la aprobación de las propuestas de nombramiento de profesorado numerario;

- la petición de informes a la Junta Acadmémica y al Claustro.

Debería configurarse como un órgano colegiado de composición no superior a los 10-12 miembros, con presencia de miembros pertenecientes a la comunidad universitaria y miembros externos a la misma. La mayoría correspondería a los miembros externos a la institución. Su Presidente sería designado por la correspondiente administración de tutela (Estado, Comunidad Autónoma), no tendría carácter ejecutivo, pero dispondría del derecho de propuesta de una parte de los Miembros externos a la institución universitaria ${ }^{9}$. El Rector es miembro nato del Consejo. El Gerente participa en las reuniones del Consejo, con voz, pero sin voto.

1.2. La Junta Académica es un órgano de consulta y propuesta que asiste ordinariamente al Consejo de Gobierno en materia docente e investigadora. Le corresponde emitir informes a petición del Consejo o por propia iniciativa. Tales informes pueden ser preceptivos, pero no vinculantes. A título de ejemplo, le corresponde informar al Consejo sobre: 
- planes de estudios y programas de investigación;

- proyectos de creación de estudios, centros o departamentos por lo que hace a su justificación académica;

- normas de admisión y permanencia de los estudiantes;

- plantillas docentes;

- propuestas de nombramiento de profesorado;

- propuestas de adscripción de centros.

Se constituiría como órgano colegiado, integrado por una representación de los responsables académicos (Decanos, Coordinadores de Estudios, Directores de Departamento e Instituto...) de las unidades de enseñanza e investigación de la Universidad. Sería presidida por el Rector.

1.3. El Consejo de Gobierno podrá también solicitar informes no vinculantes a un Claustro, constituido a partes iguales por los miembros de la Junta Académica y por una representación del personal docente, no docente y estudiantes.

1.4. El Rector es el órgano ejecutivo central de la Universidad. Como tal, corresponde al Rector:

- ejecutar los acuerdos del Consejo de Gobierno y de los demás órganos que preside;

- representar legalmente a la Universidad;

- supervisar la acción de las unidades de docencia e investigación y de los servicios administrativos y técnicos;

- presidir la Junta Académica y el Claustro;

- nombrar a Decanos, Directores y responsables de estudios y programas, a propuesta de las Comisiones mixtas correspondientes;

- proponer al Consejo de Gobierno el nombramiento de los profesores numerarios;

- proponer al Consejo de Gobierno el nombramiento y revocación del Gerente.

El Rector es nombrado por el Consejo de Gobierno entre catedráticos numerarios de Universidad. El Consejo puede revocar dicho nombramiento por mayoría cualificada de sus miembros. El mandato del Rector no será inferior a un período de seis años, renovable. El Rector puede nombrar un número limitado de Vicerrectores, para que le asistan en sus funciones ejecutivas.

2) El nivel periférico de gobierno.-Se ha dicho ya que la actividad universitaria - en docencia e investigación- se caracteriza por la autonomía que las unidades básicas o periféricas ejercen en el momento de producir y distribuir sus servicios. Pero ello no debería imposibilitar su integración en un proyecto institucional coherente ni la realización de la evaluación periódica y eficaz de sus resultados.
La organización interna de las Universidades prevista en la LRU - Centros, Departamentos e Institutos- ha evolucionado de manera diversa en las diferentes instituciones, de acuerdo con su tamaño, tradiciones, equilibrios internos de poder, etc. La reforma de los planes de estudios y el incremento de la investigación ha aumentado, si cabe, la complejidad de esta organización universitaria. Ello hace cada vez menos operativos órganos ejecutivos rígidamente basados en el criterio electivo y estamental.

Por otra parte, el conveniente equilibrio entre proyecto universitario común y descentralización de ejecución adecuada a las necesidades de cada unidad periférica puede presentar óptimos diferentes en cada institución. Por estas razones, parece lógico que cada institución reordene de manera relativamente diferenciada sus estructuras internas.

Sin embargo, por lo que hace a los órganos de gobierno de las unidades periféricas, se desprenden algunas lecciones de la experiencia común, entre las que señalamos las siguientes:

- la capacidad ejecutiva o de dirección debe confiarse a órganos unipersonales (Decanos, Coordinadores de programa, Directores de Departamento, etc.) y no colegiados;

- la designación de dichos responsables ha de ser resultado de una doble intervención: por un lado, la del colectivo/unidad encargada de las operaciones docentes o investigadoras y, por otro, la del Ejecutivo central (Rector), responsable de la supervisión de sus actividades y de la ejecución de la evaluación de las mismas. Un mecanismo de designación podría consistir en el nombramiento de tales cargos por parte del Rector, a propuesta de una Comisión de selección de candidatos, integrada por una representación de la propia unidad y una representación del propio Rector;

- esta doble intervención es la que puede asegurar el ya mencionado equilibrio entre una mayor descentralización de las responsabilidades ejecutivas y la indispensable coherencia del proyecto estratégico común de la institución universitaria. No es previsible - ni viable - un progreso en la descentralización interna sin una mayor trabazón entre los órganos ejecutivos de los niveles central y periférico;

- el mandato del responsable (Decano, Coordinador, Director, etc.) ha de tener duración suficiente para asegurar su autoridad entre sus colegas y la consolidación de su tarea. Su dedicación ha de ser reforzada con incentivos económicos y académicos;

- un Consejo de Estudios (para los programas docentes) o de Departamento (para los programas de investigación) 
dispondría de facultades consultivas para asesorar al Decano/Coordinador/Director en los aspectos docentes o científicos de su ámbito de actuación.

\section{3) Dirección politica y gestión profesional}

Está ya generalmente admitido que la complejidad de la organización universitaria actual requiere una intervención profesional, como asistencia indispensable para la dirección política. La tecnología de la gestión (financiera, informática, de infraestructuras, jurídica, recursos humanos...) no está al alcance del amateur académico. La LRU entendió acertadamente que debía asignarse a un gestor profesional la responsabilidad técnica directa de los servicios de apoyo (financieros, administrativos y logísticos) de la organización, estableciendo la figura del Gerente, incompatible con actividad académica ${ }^{10}$.

El modo de nombramiento y las funciones atribuidas a la Gerencia han sido controvertidas. En efecto, la LRU señala que el Gerente será nombrado por el Rector, oído el Consejo Social. Por otro lado, las atribuciones que se le confían lo son acon sujeción a las normas que el Consejo Social y la Junta de Gobierno establezcan, bajo la supervisión del Rectorn. A partir de ahí se han advertido posibles disfunciones en las relaciones respectivas entre Consejo Social, Junta de Gobierno, Rector y Gerencia. Por esta razón, se ha afirmado que sería más razonable una vinculación directa de la Gerencia al Consejo Social, a quien corresponden en principio las principales competencias en materia económico-patrimonial. Para ello, sería necesario que el Gerente fuera nombrado por el Consejo Social y no por el Rector.

A mi juicio, este cambio sería insuficiente -e incluso origen de mayores disfunciones-, si simultáneamente no se procede a una reasignación de competencias y redefinición de órganos. Por ello, se ha propuesto aquí la constitución de un único órgano central deliberante: el Consejo de Gobierno. A este órgano se confía la designación del Rector como principal Ejecutivo político. A su vez, correspondería al Rector el derecho de proponer al Consejo un candidato a Gerente, como apoyo profesional a su tarea ejecutiva. De este modo se facilitaría una mayor coherencia en la relación Consejo-RectorGerente.

En este esquema, el Consejo de Gobierno actuaría a modo de "consejo de administración" representativo de los intereses implicados en la institución universitaria, incluidos los de su personal. El Rector, a su vez, ocuparía la posición política de "consejero-delegado", designado por y responsable ante el mismo Consejo. Finalmente, el Gerente desempenaría la función directiva superior de carácter profesional ".

\section{Tres interrogantes finales sobre la carrera académica, la diversidad universitaria y las estrategias reformistas}

A. ¿Gobierno de la Universidad o gobierno de la profesión?

Se ha hecho referencia a la Universidad como •burocracia profesional. - en este caso, de enseñantes e investigadores-, en la que la dinámica de la corporación es tanto o más potente que la dinámica de la institución a la que los profesionales están adscritos. Esta dinámica profesional se funda en la autorregulación que los miembros de la corporación ejercen mediante la intervención en los procesos de formación, reclutamiento y promoción. Son "los iguales" - a escala nacional e internacional- quienes ejercen el control más efectivo sobre el funcionamiento de la corporación.

Por su parte, la capacidad de incidencia de la institución es débil. Depende de la acción de los propios profesionales que —en gran medida - se autoorganizan, prescindiendo a menudo de la institución en que prestan sus servicios y sintiéndose más identificados con el colectivo profesional que los evalúa y promociona.

Pero este desajuste entre lógica de la corporación y necesidades de la institución tiene efectos negativos cuando se hace más aguda la presión -social, política y económicasobre la institución universitaria, como proveedora de servicios a la sociedad. La gobernabilidad institucional $\longrightarrow$ capacidad de adaptación de la universidad a las exigencias de su entorno- está lastrada —o bloqueada — por las lealtades corporativas.

Es razonable preguntarse por tanto en qué medida es posible una mayor gobernabilidad institucional si sólo se modifican sus mecanismos internos de decisión y no se reforma paralelamente el modelo de gestión/gobierno de la profesión. Algunas reformas en este aspecto se han puesto ya en marcha en algunos países y se debaten en otros: cambios en la naturaleza y características de la relación laboral (nombramientos vitalicios, estatuto funcionarial), sistemas de reclutamiento, promoción y movilidad, esquema de retribuciones e incentivos, etc. Compartimos el punto de vista de quienes consideran que la reforma del gobierno de la institución universitaria sólo puede tener efectos consistentes si se acompaña con una revisión del gobierno de la profesión ${ }^{12}$. 
b. ¿Modelo único de gobierno o diversidad de modelos?

La segunda observación arranca de la duda sobre la persistencia de un modelo único de "Universidad. ¿Es válido todavía hablar de ala" Universidad? ¿O hay que admitir que el término recubre hoy realidades muy diferenciadas en objetivos, tradiciones, dimensiones, contextos socioculturales, etc.?

En tal caso, la pretensión de fijar un patrón único para la ordenación de los órganos de gobierno de las Universidades sería inútil e incluso perjudicial. Es cierto que existe una conciencia extendida sobre el funcionamiento deficiente - en España y en otros países- del autogobierno universitario y que se dan factores comunes, como puede ser la existencia de fuertes inercias corporativas resistentes a la renovación. Pero la heterogeneidad de la realidad universitaria actual hace difícil afirmar la existencia de una misma receta para todas las instituciones.

Por ello, no habría que descartar la previsión de modelos alternativos de gobierno, a los que pudieran acogerse las instituciones en función de la diversidad de sus características. Del mismo modo, sería útil explorar la vía de modelos experimentales, que permitieran luego contrastar resultados.

\section{C. ¿Qué estrategia para la reforma?}

No es éste el lugar adecuado para tratar a fondo de la estrategia más útil para hacer frente a los problemas señalados. Con todo, es fácil admitir que una estrategia exclusivamente normativista - una mera reforma legal— puede fracasar fácilmente, si no va acompañada de otras actuaciones. Entre ellas cabe señalar, en primer lugar, la aplicación de incentivos positivos y negativos a las conductas institucionales e individuales. La asignación de recursos por parte de las administraciones de tutela puede ser un instrumento efectivo en un proceso de reforma.

En segundo lugar, sin una presión exterior de cierto impacto tampoco parece fácil una rectificación del statu quo: priman con frecuencia las posturas de bloqueo y veto sobre las de cambio e innovación. Algunos representantes externos en los Consejos Sociales pueden - a partir de su experiencia- trasladar a la institución las razonables expectativas del entorno y promover iniciativas de revisión de los aspectos menos eficientes y más opacos del gobierno universitario.

Finalmente, no puede ignorarse la resistencia de las propias administraciones de tutela -Ministerio, Consejerías autonómicas- a abordar adecuadamente un asunto que comporta rendimientos políticos negativos ante determinados sectores de lia opinión. Estos "costes" pueden ser mejor asumidos si se consiguen articular las necesarias coaliciones entre agentes sociales; sectores académicos y responsables políticos, cuya visión podría trascender el inmediatismo del ciclo electoral. Esto permitiría ensayar, aunque fuera de forma parcial y a título experimental, fórmulas que rectificaran las deficiencias actuales y que permitieran la disminución de sus efectos negativos sobre el rendimiento social exigible a un sistema universitario público.
- Catedrático de Ciencia Politica y de la Administración. Departament de Ciència Política i Dret Públic. Universitat Autònoma de Barcelona.

1 Este texto corresponde a la comunicación presentada en el Seminario sobre gobierno universitario, organizado en Ciudad Real por el Consejo de Universidades, la Fundación Universidad-Empresa y la Universidad de Castilla-La Mancha en junio de 1996. Un desarrollo de las ideas aqui expuestas se ofrece en VALLĖS, J. M. (1995), Governabilitat i Universitat puiblica: anàlisi i proposta. Escola d'Administració Pública de Catalunya, Barcelona.

2 El alcance internacional del problema ha sido puesto de relieve en estudios comparados sobre la enseñanza superior, como el Informe recientemente publicado por la UNESCO. En dicho Informe se señala muy explicitamente: •Due attention should be paid to the observance of the principles of academic freedom and institutional autonomy. However, those principles should not be invoked in order to militate against necessary changes or as a cover for narraowly interpreted corporatist attitudes and abuse of privileges that can, in the long run, have a negative effect on the functioning of higher education. (UNESCO, 1995, Policy Paper for Change and Development in Higher Education, Paris: 9, 26).

${ }^{3}$ Dejamos de lado otros aspecios centrales de la LRU, como puede ser la opción por la funcionarización del profesorado o el bien conocido artículo 11. No puede ignorarse su impacto sobre el asunto que comentamos en esta nota, aunque no nos ocupemos de ellos.

${ }^{4}$ Cfr. ClaRK, B. R. (1983), The Higher Education System: Academic Organisation in Cross-Cultural Perspective. University of California Press, Berkeley; Gofdegfruure,
L. et alt (1993), Higher Education Policy. An International Comparative Perspectiv'. Oxford, Pergamon Press.

' Recordemos que ministros, secretarios de Estado, directores generales, consejeros autonómicos, etc., responsables de la política universitaria suelen pertenecer : la profesión docente o universitaria. Atribuir a una cabstracta. administración la sola responsabilidad de aquellas decisiones es ignorar la permanente intervención qui un sector de la propia profesión ha ejercido tradicionalmente y sigue ejerciendo en la orientación de la política universitaria de la mayoría de los paises, ya sea como expertos consultores, ya sea como operadores políticos o ejecutivos.

6 Aunque comparten generalmente una misma lógica corporativa, se dan algunas diferencias de comportamiento en los tres estamentos*, reflejadas en la intensidacl de su participación en la elección de representantes en los órganos colegiados, ('n la articulación asociativa de dichos representantes - sindical, parasindical " parapolítica, cultural, etc.-, en el grado de intervención en las deliberaciones dk. los órganos de gobierno o en la disciplina de voto que observan. No podemos desarrollar aquí estas diferencias.

' Cfr. MintzBeRG, H. (1984), La estructuración de las organizaciones. Ariel, Barcelona.

8 Para un desarrollo más detallado, cfr. Vallès, o.c.

9 Con ello se pretende reforzar el papel del Presidente - sin convertirlo en cargon ejecutivo-y, simultáneamente, se busca evitar alguna deriva corporativa que podria afectar también al propio Consejo, tal como se ha experimentado en algunos Consejos Sociales. 
10 No parece acorde con la idea original de la LRU la práclica de designar como Gerente a un profesor de la propia Universidad, a veces sin gran experiencia de gestión en organizaciones complejas y sin vocación de permanencia en una carrera gerencial.

"No se trata aquí de la configuración de la gestión profesional en las unidades periféricas. Sólo cabe indicar que el órgano ejecutivo políico-académico de estas unidades requiere también la asistencia de una figura profesional de nivel directivo.

1: Cfr. sobre este aspecto los trabajos de FredBerg, E.-Musseux, C. (1987), Marcbé des Professeurs et Integration Institutionnelle, CNRS, Paris. También de los mismos autores (1989), En quête d'Université. L'Harmattan, Paris. 
